\title{
Verification of Electromagnetic Field Measurements via Inter-laboratory Comparison Measurements
}

\author{
M. Mann ${ }^{1}$, H. Brüggemeyer ${ }^{2}$, and P. Weiß ${ }^{1}$ \\ ${ }^{1}$ University of Kaiserslautern, Department Of Electrical Engineering, Chair Of High-Voltage-Engineering And EMC, \\ P.O. Box 3049, 67663 Kaiserslautern, Germany \\ ${ }^{2}$ Lower Saxony Federal State Trade Supervisory Office, Dr. H. Brüggemeyer, Göttingerstr. 14, 30449 Hannover, Germany
}

\begin{abstract}
An inter-laboratory comparison of field strength measurements was conducted in order to verify the comparability of high-frequency electromagnetic field measurements. For this purpose, 17 participating teams hosted by the working group "procedures of exposure determination" of the LAI (Länderausschuss für Immissionsschutz, state committee on immission control) determined the field strength at given stations around a hospital situation. At those stations very different signals were generated, such as sine wave signals at $27 \mathrm{MHz}$ and $433 \mathrm{MHz}$, signals from a diathermy device in Continuous-Wave (CW) and PulseWidth-Modulation (PWM) mode, from a GSM base station at $900 \mathrm{MHz}$ and $1800 \mathrm{MHz}$, from a UMTS base station, from a babyphone device and from a DECT cordless phone. This contribution describes the evaluation of the measured values and the approach to the computation of a reference value. Considering various sources of electromagnetic fields in the areas of personal safety at work and of immission control, the most important results are presented and the conclusions drawn are discussed.
\end{abstract}

\section{Introduction}

Reproducible electromagnetic field measurements may create and convey credibility to the public. Solely measurements of adequate quality are able to put an evaluation of personal exposure on a firm basis. The revisable quality of the measurement procedure and instrumentation is the crucial factor to achieve good results. In the field of safety at work, e.g. employees working at high-frequency (HF) transmitter stations entrust their life to the results of electromagnetic field strength measurements.

An appropriate measurement system and the traceability of measurement results to the SI units are prerequisites for credible measurements. In order to keep the quality on the highest level possible, a suitable Quality Assurance (QA) system being implemented at each testing or measurement facility is necessary. Amongst other things, QA consists of

Correspondence to: $\mathrm{M}$. Mann

(michael.mann@eit.uni-kl.de) the documentation of the employees' mandatory qualification and the adopted measures to sustain this qualification.

The instruments applied for verification and measurement, are to be revised by internal and external calibration and supervision at regular intervals with respect to precision, accuracy and a reasonable representation of the measured quantity by the indicated value. The external evaluation of accuracy is usually verified by inter-laboratory comparison measurements checking both equipment and personal qualification simultaneously.

\section{Objective of the inter-laboratory comparison}

The objective of this inter-laboratory comparison is to find the deviation in results, when different teams measure the RMS-value of the electrical field strength within the same area or setup. The measurements were performed at test setups derived from real-life situations, not in anechoic chambers, where artificial and constant ambient conditions can be assured at all times. Some of the tests were even carried out at outside measuring points, where environmental conditions were expected to alter over the period that the tests took place.

In order to ensure the comparability of official highfrequency electromagnetic field measurements in practical applications, the inter-laboratory comparison was hosted in May 2003 by the working group "procedures of exposure determination" of the LAI (Länderausschuss für Immissionsschutz, state committee on immission control). In contrast to other inter-laboratory comparisons, the measuring devices and measuring procedures should be qualified, rather than qualifying the measuring teams.

\section{Implementation of the inter-laboratory comparison}

\subsection{Traceability to national measurement standards}

Traceability of physical quantities to SI units means that measurements are taken in an unbroken chain with calibrated measurement equipment and with known uncertainty for each step. Therefore, most of the countries operate a 
Table 1. Evaluation of reference measurement "sine wave $27 \mathrm{MHz}$ “.

\begin{tabular}{|c|c|}
\hline \multicolumn{2}{|l|}{ measuring point "sine wave at $27 \mathrm{MHz}$} \\
\hline mean value $[\mathrm{V} / \mathrm{m}]$ & 2,29 \\
\hline standard deviation of mean values $[\mathrm{V} / \mathrm{m}]$ & 0,21 \\
\hline max (standard deviation of measuring cycles M1 up to M6) [V/m] & 0,07 \\
\hline linear regression & \\
\hline relevant frequencies: $\mathrm{f} 1[\mathrm{MHz}]$ & 26,971 \\
\hline extrapolated calibration factor of probe & \\
\hline position PE [ ] & 0,89 \\
\hline position $\mathrm{PH}[$ & 1,03 \\
\hline position PS & 1,03 \\
\hline minimal calibration factor of probe [] & 0,89 \\
\hline maximal calibration factor of probe [] & 1,03 \\
\hline best estimate of calibration factor of probe [ ] & 0,96 \\
\hline relative standard deviation of calibration factor of probe [ $\pm \%]$ & 0,07 \\
\hline calibration field at PTB: relative standard deviation GTEM-cell $(1 \sigma)[ \pm \%]$ & 0,06 \\
\hline mean values: maximal relative standard deviation [ $\pm \%]$ & 0,10 \\
\hline relative standard deviation of reference value $(1 \sigma)[ \pm \%]$ & 0,14 \\
\hline relative expanded measurement uncertainty of reference value $(2 \sigma)[ \pm \%]$ & 0,27 \\
\hline relative expanded measurement uncertainty of reference value $(2 \sigma)[ \pm \mathrm{dB}]$ & 2,09 \\
\hline referece value: minimum $[\mathrm{V} / \mathrm{m}]$ & 1,60 \\
\hline reference value: best estimate $[\mathrm{V} / \mathrm{m}]$ & 2,20 \\
\hline reference value: maximum $[\mathrm{V} / \mathrm{m}]$ & 2,80 \\
\hline
\end{tabular}

national metrology institute. Tasks of PTB (PhysikalischTechnische Bundesanstalt, the national metrology institute of Germany) are the determination of fundamental and natural constants, the realization, maintenance and dissemination of the legal units of the SI, safety engineering, services and metrology for the area regulated by law and for industry.

In practice, the generation of the basic quantities of electromagnetic fields - electric $(E)$ and magnetic field strength $(H)$ and power flux density $(S)$ respectively - require a calculable transmitter, a calculable receiver or a suitable transfer probe, which is calibrated at a "standard measuring equipment". For the inter-laboratory comparison described herein, traceability is obtained by the application of a "reference transfer probe" that was calibrated at PTB.

\subsection{Evaluation of measurements}

In principle, there are two methods of determining a best representation of a measurand. On the one hand, by the application of measuring devices traced directly to national measurement standards via a calibration with the lowest possible uncertainty, on the other hand via averaging a multitude of measured values after excluding outliers. Both procedures were applied in the context of the evaluation at hand.

The exact value of the electric field strength $E$ is unknown at any time for the inter-laboratory comparison described herein, only the best estimate for $E$ can be obtained. In theory, it can be calculated on the basis of transmitted power, the directivity of the antenna and the law of squared distance for regions free of reflection at far-field conditions:

$E_{\text {eff }}=\sqrt{\frac{P_{0} \cdot Z_{0} \cdot G_{\text {linear }}}{4 \cdot \pi \cdot r^{2}}}$

$P_{0}$ designates the power of the transmitter and $Z_{0}$ is the characteristic impedance of free space. $G_{d B}$, transformed to linear scale $G_{\text {linear }}$, represents the accumulated gain of the transmitting antenna, the attenuation of the feeding cable and the horizontal and vertical attenuation, which can be determined from the horizontal and vertical antenna diagram. The distance between measuring point and the position of the antenna is referred to as $r$. In practice, the reflection and attenuation phenomena that will occur will render the calculation of nearly exact nominal values according to Eq. (1) difficult since environmental conditions (e.g. ground conductivity, weather conditions) alter. The electric field strength may vary considerably with respect to the position of the probe and the time of the measurement. Hence, it can only be measured.

A broadband measuring system was applied for reference measurement cycles. The results were utilized for the calculation of "reference values". An estimate of the best representation of a measurand may be gained using a probe 
with its calibration traced directly to national measurement standards providing the lowest possible measurement uncertainty. The calibration of a measuring device allows us to determine the deviation of the indicated value from the nominal value of the measurand. It is obvious, that results obtained with a traceably calibrated probe give a better representation of the actual value of the physical quantity to be obtained. The construction of the probes limits the accuracy of measurements despite a traceable calibration.

One reference measurement cycle consists of the recording of 50 up to 350 individual values at a time interval of $0.4 \mathrm{~s}$ up to $2 \mathrm{~s}$. Up to seven measurement cycles were completed at each measuring point. The arithmetic mean and the standard deviation were calculated for the recorded values of each measurement cycle. The subsequent calculation of the total uncertainty is based on these average values.

The participating teams - composed of both official and non-official institutions commissioned to field strength measurements - were asked to report the total uncertainty of each measured value. The uncertainty reported by each of the participating teams is compared with the total uncertainty of the reference values in order to gain insight into the stability of the respective transmitter.

\subsection{Uncertainties of reference values}

In order to be able to qualify the measurement procedures, the calculation of the uncertainty of each reference measurement was compulsory.

The evaluation of the reference measurement is exemplified for the measuring point "sine wave $27 \mathrm{MHz}$ " (Table 1). The "standard deviation of mean values" represents the uncertainties resulting from inaccurate positioning of the measurement device at repetitive measuring cycles, in short: repetition accuracy. Each measuring cycle is considered stochastically independent.

In addition to the standard deviation of the mean values of the measurement cycles M1 up to M6, the maximum value of the standard deviation of each measurement cycle is calculated.

The bound of $\pm 1 \sigma$ (coverage factor $k=1$ ) of a Gaussian variable is referred to as standard uncertainty, the bound of $\pm 2 \sigma(k=2)$ as expanded measurement uncertainty. The probability of a Gaussian variable being within the confidence interval is $95 \%$ for a coverage factor of $k=2$.

Instable transmitters may fluctuate at varying periods. If a transmitter fluctuates at a period that is long in comparison to the duration of a measuring cycle, the resulting uncertainty is represented by the "standard deviation of mean values". However, if the transmitter has a period of fluctuation, which is very short compared to the duration of a measuring cycle, the "standard deviation of the measuring cycle" will alter. The "uncertainty of measuring cycles" represents the noise of the measurement devices and transmitters. The "uncertainty of mean values" is not correlated with the "uncertainty of measuring cycles". Hence, the standard deviations are to be added by the root-sum-square (RSS) method. This circumstance is apparent in Table 1: The maximum "standard deviation of measuring cycles" constitutes $\pm 0.07 \mathrm{~V} / \mathrm{m}$ for the measuring point "sine wave $27 \mathrm{MHz}$ "; a fraction of the "standard deviation of mean values", which is $\pm 0.21 \mathrm{~V} / \mathrm{m}$. We can conclude that the transmitter is comparatively stable, the total uncertainty is mainly constituted by the reproducibility of the measuring cycles.

Vice versa, the "standard deviation of mean values" $( \pm 4.36 \mathrm{~V} / \mathrm{m})$ is merely $40 \%$ of the maximum "standard deviation of measuring cycles" $( \pm 10.68 \mathrm{~V} / \mathrm{m})$ in case of the diathermy device in PWM mode. From this it follows that the total uncertainty is primarily caused by the instability of the transmitter.

Another cause of uncertainty is the anisotropy of the electromagnetic field probe. The calibration certificates of the reference measurement devices provide calibration factors, which were extrapolated to the emitted frequencies by linear regression. The application of an isotropic field probe with diode detector renders the allocation of the detected field strength to a certain frequency or a certain direction impossible. Thus, the calibration factors are calculated for each emitted frequency and each direction. The best estimate of the calibration factor is centred between the minimum and maximum value of the extrapolated calibration factors. The difference between best estimate and extreme values represents the uncertainty $(k=1)$ of the calibration factor at the current measuring point.

The total value of the standard deviation is obtained by geometrical addition (i.e. RSS) of the previously discussed values (ISO, 1995). The best estimate of the reference field strength can be determined by multiplying the mean value with the best estimate of the calibration factor.

One basic approach of Quality Assurance may consist of limiting the total value of uncertainty. At Switzerland (BUWAL, 2002) for example, the expanded measurement uncertainty is limited to $\pm 45 \%$ ( $\pm 3.2 \mathrm{~dB}$ ) for GSM mobile telephone base stations. Defining such a limit requires a thorough consideration with regard to the capabilities of the measurement devices and the signal to be measured.

The expanded measurement uncertainty of the national measurement standard for the generation of an empty field adds up to $\pm 12 \%$ ( $\pm 1 \mathrm{~dB}$ ) for the given example (GTEMcell, $f<1 \mathrm{GHz})-$ i.e. for most of the isotropic broadband measuring systems applied. Considering this, it would be unrealistic to specify the total value of the expanded measurement uncertainty for a device calibrated by this standard to be $\pm 6 \%( \pm 0,5 \mathrm{~dB})$.

\subsection{Discussion of the $\mathrm{E}_{N}$-value}

In order to compare the measured value of a participating team with the reference value, the so-called $\mathrm{E}_{N}$-value (EAL, 1994) is applied. This scalar value allows the comparison of the results of each team and the reference value with simultaneous consideration of all relevant uncertainties. The 


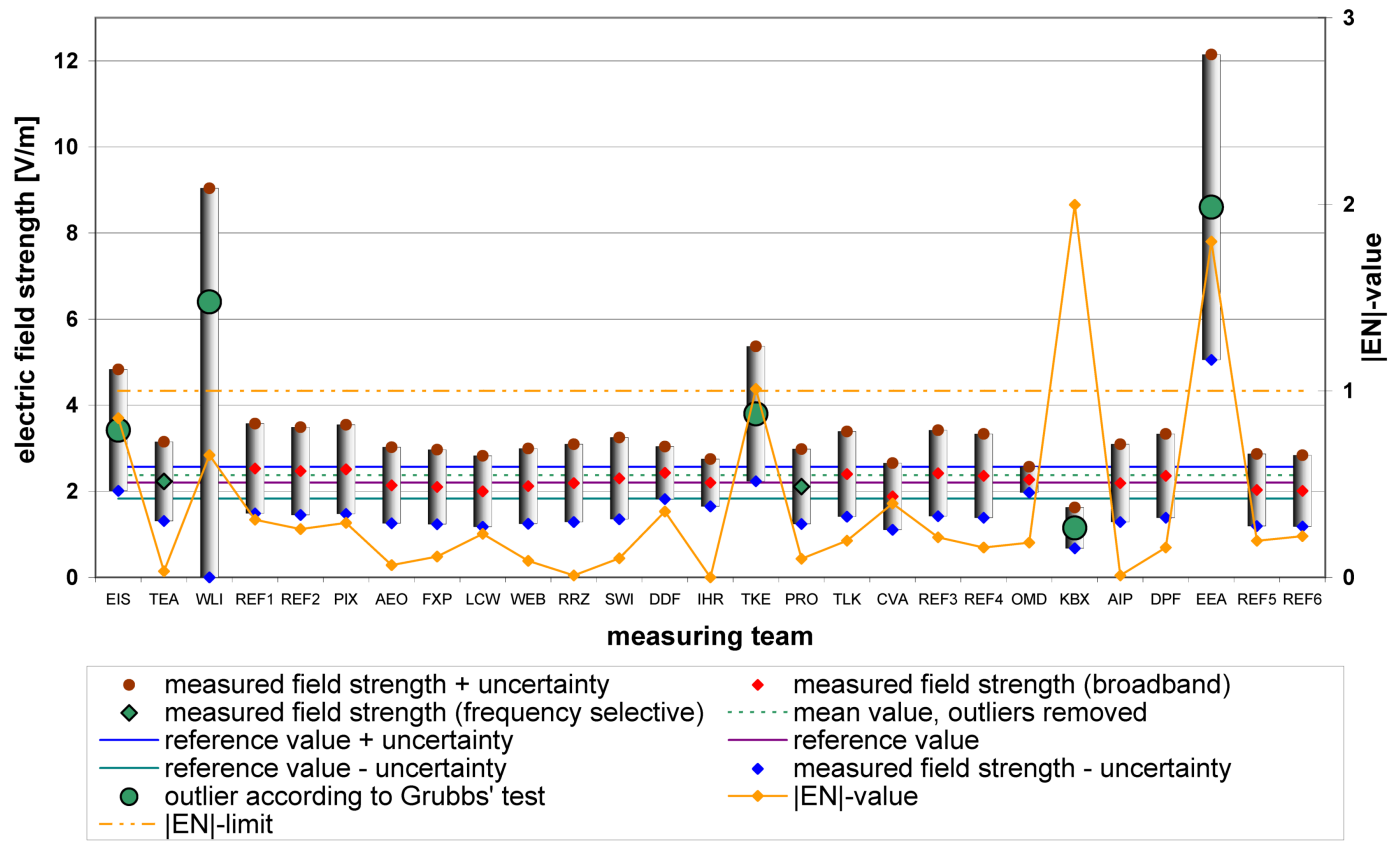

Fig. 1. Evaluation of the measuring point "sine wave $27 \mathrm{MHz}$ ".

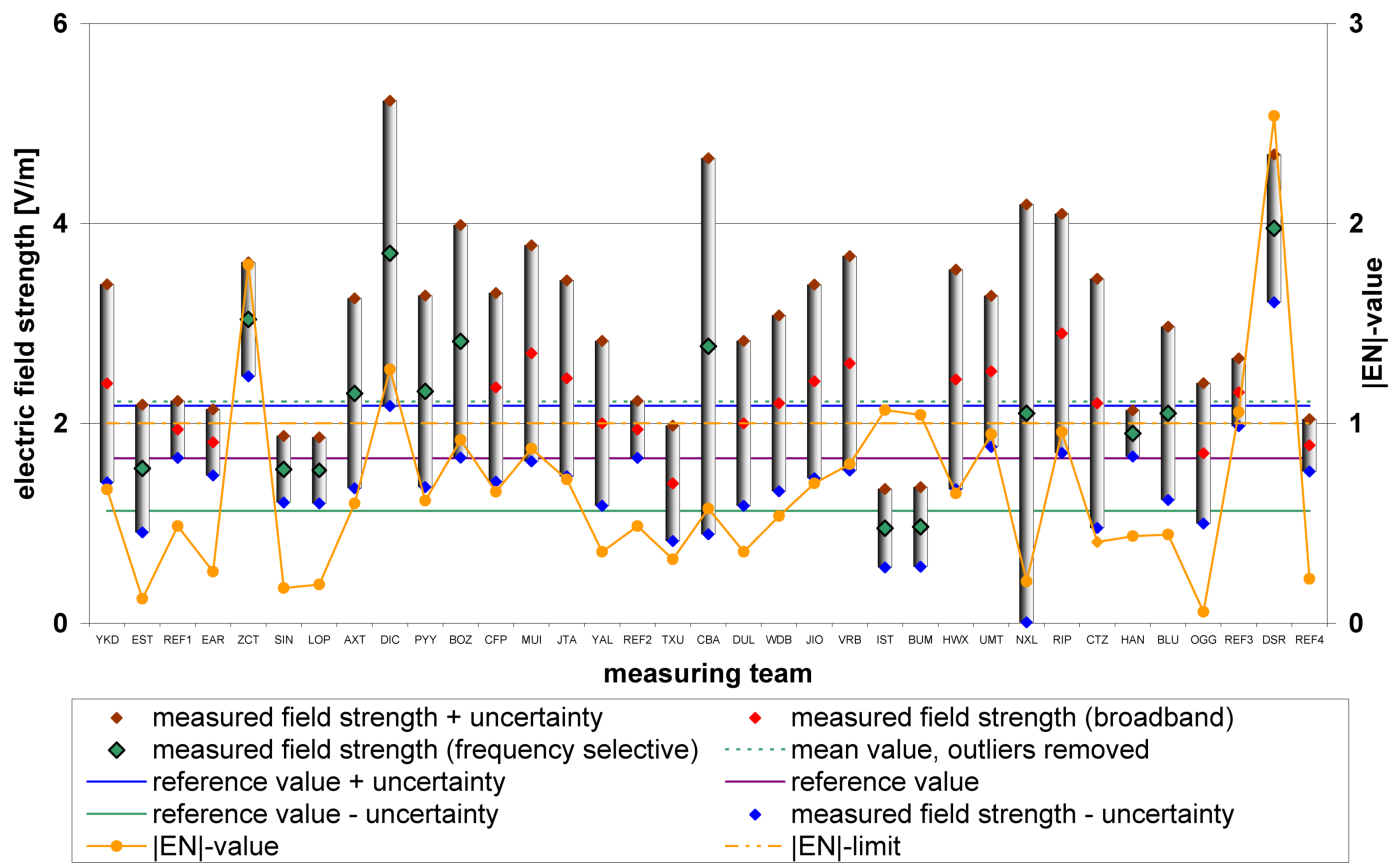

Fig. 2. Evaluation of the measuring point "UMTS".

$\mathrm{E}_{N}$-Value of a measurement is determined as follows:

$E_{N}=\frac{\left|X_{l a b}-X_{r e f}\right|}{\sqrt{U_{l a b}^{2}+U_{r e f}^{2}}}$

$X_{l a b}$ indicates the measured value of a team, $X_{r e f}$ the reference value, $U_{l a b}$ the expanded measurement uncertainty of the team, $U_{\text {ref }}$ the expanded measurement uncertainty of the reference measurement at a given measuring point.
The $\mathrm{E}_{N}$-value does not allow for conclusions in terms of accuracy or the quality of the teams among one another. For $\mathrm{E}_{N}<1$, the measured results are assessed to be credible, for $\mathrm{E}_{N}>1$, the results are regarded as questionable. The level of credibility decreases with the increase of the $\mathrm{E}_{N}$-value. 


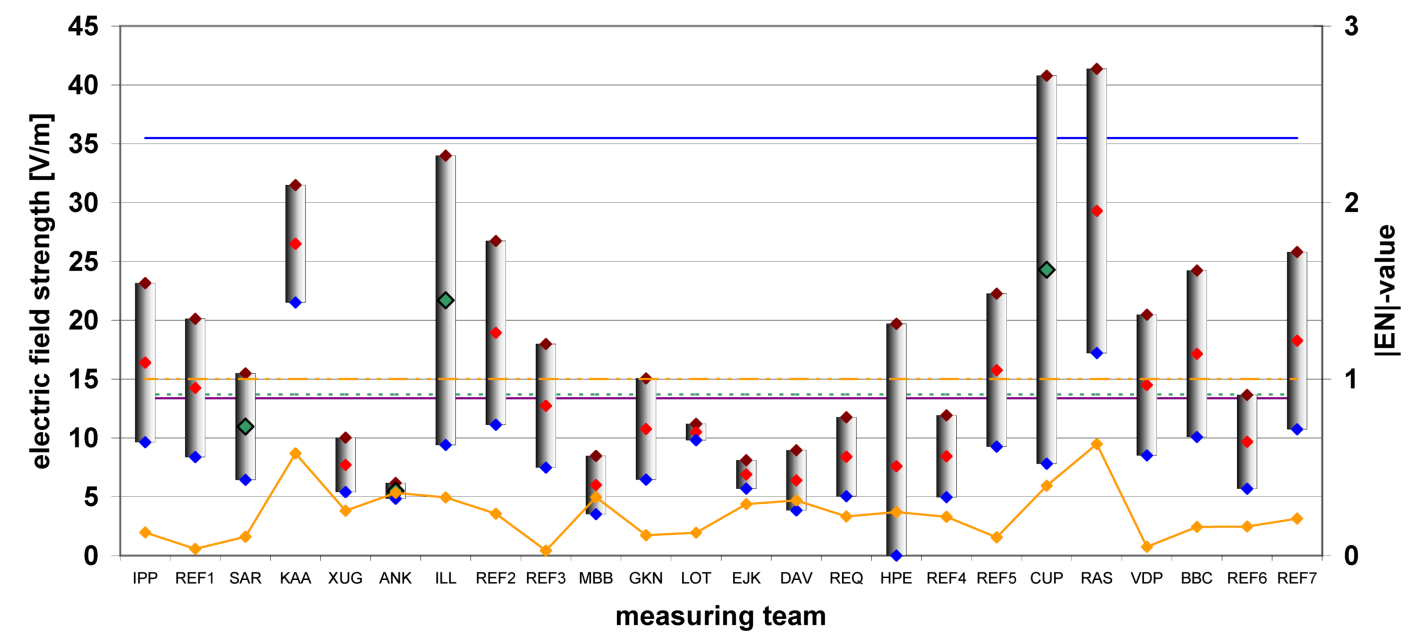

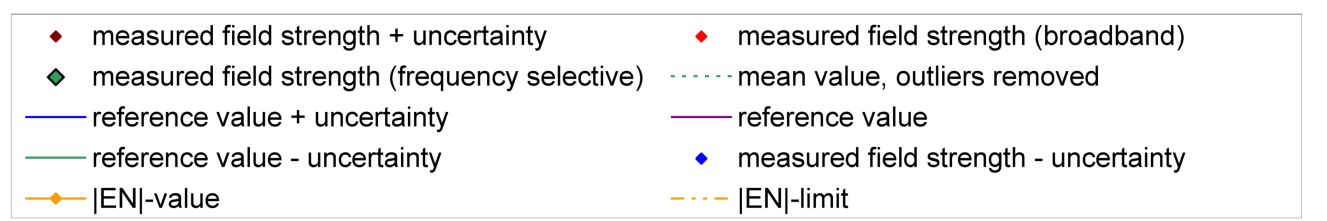

Fig. 3. Evaluation of the measuring point "diathermy device in PWM mode".

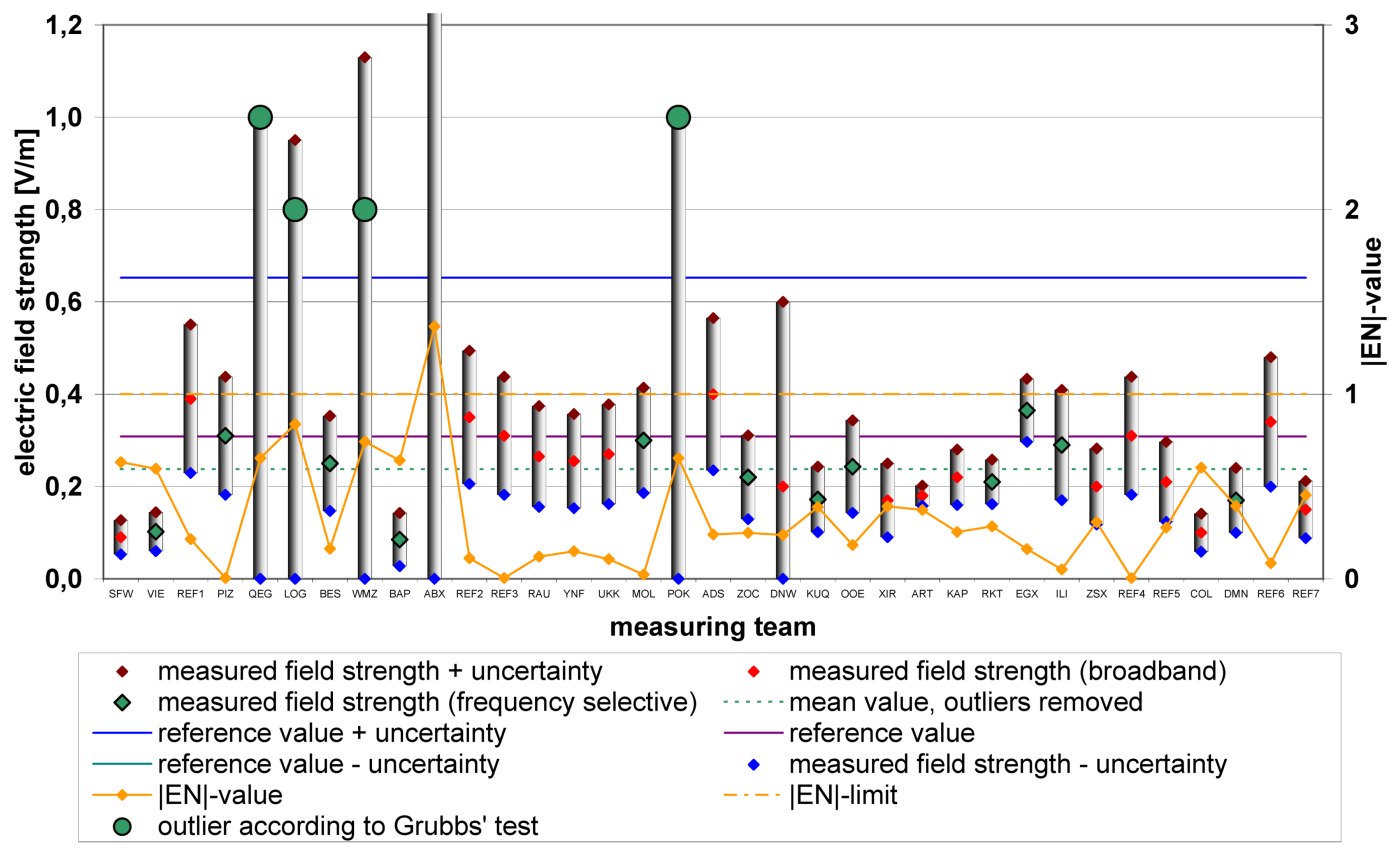

Fig. 4. Evaluation of the measuring point "Babyphone".

\section{Results of the inter-laboratory comparison}

\subsection{Sine Wave $27 \mathrm{MHz}$}

The allocation of measured values to measuring teams used a randomised three-letter code. The diagrams of the evaluation show the measured values versus the team codes including the relevant uncertainty values in chronological order (Fig. 1). The results of the reference measurements were labelled REF1 up to REFn, where $\mathrm{n}$ is the number of measuring cycles performed applying the "reference transfer probe". The result of each "reference measurement cycle" was calculated without the application of the frequency-dependent calibration factor.

Yet, the average of the reference measurements was multiplied with the best estimate of the calibration factor to generate the "reference value". This may lead to visible variations 
Table 2. Summary of the results.

\begin{tabular}{|c|c|c|c|}
\hline $\begin{array}{l}\text { measuring } \\
\text { point }\end{array}$ & frequency & $\begin{array}{l}\text { mean } \\
\text { value }\end{array}$ & $\begin{array}{l}\text { reference } \\
\text { value }\end{array}$ \\
\hline & [MHz] & {$[\mathrm{V} / \mathrm{m}]$} & {$[\mathrm{V} / \mathrm{m}]$} \\
\hline sinus & 27 & $2,34 \pm 0,75$ & $2,20 \pm$ \\
\hline UMTS & 2167 & $2,12 \pm 1,28$ & $1,65 \pm$ \\
\hline diathermy PWM & $2450 \pm 50$ & $13,69 \pm 13,54$ & $13,37 \pm 22,12$ \\
\hline Babyphone & 434 & $0,24 \pm$ & $0,31 \pm$ \\
\hline
\end{tabular}

between the measured values of the reference probe and the averaged reference value.

Considering all measured values of the field strength at a certain measuring point, the mean value was calculated after applying a Grubbs' test for outliers with a confidence interval of $95 \%$ (Adunka, 2000). The values identified as outliers were disregarded for the calculation of the mean value. The averaging process did not weight the reference measurement in any way.

The subsequent example illustrates the necessity of a test for outliers prior to the calculation of the mean value. If the measured value of a fictive measurement device was constantly set to $X_{l a b}=0$, and the uncertainty was $U_{l a b}=\infty$, then the $\mathrm{E}_{N}$-value would be $\left|E_{N}\right|=0$ solely due to the high uncertainty. Thus, without a test for outliers, an undetected outlier would affect the mean value. Generally speaking, measurements possessing a high value of uncertainty are not qualified for the accurate determination of exposure to electromagnetic fields. Measurements possessing a high value of uncertainty were not excluded from the calculations a priori. However, if teams generated results that were identified as outliers later, they were excluded from the calculation of the mean value for the respective measuring point.

\subsection{UMTS}

Regarding compliance measurements with frequencyselective measurement devices, the request for a minimum resolution bandwidth of $\mathrm{RBW} \geq 5 \mathrm{MHz}$ is coupled with the request for choosing an appropriate detector envelope. According to regulations, the RMS detector has to be applied. Some teams presented results gained by consciously incorrect application of the Max-Peak detector (Fig. 2).

An overvaluation of twice the amount can be observed comparing the teams DIC (8th team from the left, Max-Peak) with AXT (9th team from the left, RMS), which leads to an increase in the statistical spread.

\subsection{Diathermy device in PWM mode}

The uncertainty bound of $\pm 165 \%$ represents an almost statistical distribution of the measured values (Fig. 3). The socalled "PWM mode" challenged the teams due to its instability and inaccuracy. Should a measurement with a low uncertainty be imperative, extensive measurements at low metering time periods have to be conducted.

\subsection{Babyphone}

Broadband measuring instruments derived from the field of safety at work were designed to validate the compliance to limit values. Originally, they were not designed to be sufficiently sensitive to measure field strength levels well below $1 \mathrm{~V} / \mathrm{m}$. According to the probe in use, the measurement shows a specific threshold level due to the noise floor. In case of the probe applied for the reference measurements, this threshold level amounts to $E=0.6 \mathrm{~V} / \mathrm{m}$. Results below this level are not considered to be qualified. This experience was to be validated by the participating teams (Fig. 4).

The "Babyphone" measuring point is of interest because the respective devices are subjected to public criticism in spite of having a relatively low power output. The results are not expected to be exact due to the inhomogeneity of the field, the inaccurate positioning of probes and the distance between the probe and the transmitter antenna of approximately $0.1 \mathrm{~m}$. One can merely state that the field strength is below $0.6 \mathrm{~V} / \mathrm{m}$ due to the noise floor of the reference probe.

\section{Summary and prospect}

The results of the inter-laboratory comparison show a significant deviation of the uncertainty values determined for each measuring point (Table 2).

The inter-laboratory comparison indicated that the measurement devices are generally capable of validating the compliance with legal limit values if the employees have the required know-how. Furthermore, a realistic estimation of the total uncertainty is essential considering the uncertainty of the measuring devices, the uncertainty of the sampling and the parameters of the transmitter. Considering the technological limits, the comparability between broadband and frequency-selective measurement devices is given. One should be aware of the limited capabilities of broadband measurement devices. CISPR 16-1 specifies the compulsory application of frequency-selective measurements employing a quasi-peak detector at radio disturbance measurements close to the limit value of the field strength (CISPR, 1997). Isotropic measurement is more complex if frequency-selective devices are applied.

In general, the sensitivity of broadband devices is not sufficient for an accurate detection of low field strength values in public areas. Broadband devices are hardly applicable to determine the field strength of complex signals (e.g. diathermy device in PWM mode).

Frequency-selective measurements permit the separation and identification of multiple transmitter stations. The field strength of multiple sources can be compared to the frequency-dependant limit values. Hence, the exposition can be assessed more accurately in comparison to broadband devices. An improved inter-laboratory comparison concept 
would employ a frequency-selective reference measurement device. Participation of as many teams as possible would broaden the statistical base, but only teams providing a small uncertainty should be involved.

Continuous QA can provide measurements of adequate accuracy. Repetitive inter-laboratory comparisons constitute an essential part of QA systems.

\section{References}

Adunka, F.: Messunsicherheiten, Theorie und Praxis, Vulkan Publishing, Essen, Germany, 2000.

Bundesamt für Umwelt, Wald und Landschaft (BUWAL): Mobilfunkbasisstationen (GSM) - Messempfehlung, Bern, Switzerland, 2002.

Comité International Spécial des Perturbations Radioélectriques: CISPR 16-1, Specification for radio disturbance and immunity measuring apparatus and methods, Part 1: Radio disturbance and immunity measuring apparatus, July 1997.

European Cooperation for Accreditation of Laboratories (EAL): EAL Inter-laboratory Comparisons, 1996.

International Organization for Standardization: Guide to the Expression of Uncertainty in Measurement, 1st Edition, 1993, corrected and reprinted 1995, Geneva, Switzerland, Beuth Publishing, Berlin, Wien, Zürich, 1995. 